\title{
Metabolic footprinting revealed key biochemical changes in a brown fermented milk product using Streptococcus thermophilus
}

\author{
DanYang Li, ${ }^{1,2 *} \odot$ Yan Zheng, ${ }^{1,2 *}$ Lai-yu Kwok, ${ }^{1,2} \odot$ WenYi Zhang, ${ }^{1,2} \odot$ and TianSong Sun ${ }^{1,2} \dagger$ \\ ${ }^{1}$ Key Laboratory of Dairy Biotechnology and Engineering, Ministry of Education P. R. C., Inner Mongolia Agricultural University, Hohhot 010018 , \\ P. R. China \\ ${ }^{2}$ Key Laboratory of Dairy Products Processing, Ministry of Agriculture and Rural Affairs P. R. C., Inner Mongolia Agricultural University, \\ Hohhot 010018, P. R. China
}

\begin{abstract}
Ultra-performance liquid chromatography coupled with a quadrupole-time-of-flight mass spectrometry ${ }^{\text {Elevated Energy }}$ was used to investigate changes in the metabolite profile of brown milk and fermented brown milk produced using Streptococcus thermophilus S10. Samples were analyzed in both positive and negative electron ionization modes. Data were analyzed by multivariate statistical methods for biomarker metabolites that were differentially abundant in brown milk and fermented brown milk. We identified 43 differentially abundant metabolites based on mass spectrophotometry fragmentation patterns. These metabolites included peptides, AA, fatty acids and related metabolites, carbohydrate metabolites, vitamins, and nucleosides. Some of these metabolites are known to alter the sensorial quality of fermented dairy products. Thus, it is likely that some of the currently identified differentially abundant metabolites also contribute to the unique flavor, taste, and aroma of fermented brown milk. The bitterness and astringency of fermented brown milk are likely to be due to some of these peptides, whereas the sweetness and sourness could be a result of changes in carbohydrate levels. No previous study has analyzed metabolomics changes during fermentation of brown milk. Thus, our data are a valuable reference for future development and improvement of fermented brown milk products.
\end{abstract}

Key words: fermented brown milk, Streptococcus thermophilus, ultra-performance liquid chromatography coupled with a quadrupole-time-of-flight mass spectrometry with elevated energy, metabolite

Received April 29, 2019

Accepted November 18, 2019 .

*These authors contributed equally to this work.

†Corresponding author: sts9940@sina.com

\section{INTRODUCTION}

Fermented milk has been consumed for centuries and is popular for its nutritional value and appealing flavor. The process of fermented milk production is based on the acidic destabilization of milk proteins by thermophilic lactic acid bacteria, which is facilitated by optimum heat-induced protein denaturation (Loveday et al., 2013). A variety of fermented milk products are available in the commercial market, including traditional yogurt and Greek yogurt. In China, fermented brown milk has emerged as a new product in the past $2 \mathrm{yr}$, with a variety of different forms; these include cooked fermented milk and Russian-style char-grilled fermented milk (Ryazhenka; Ma et al., 2016). Fermented brown milk differs from traditional products in terms of taste and color, which is due to the manufacturing process. In contrast to traditional yogurt and other products, fermented brown milk is produced by incubating the milk at a high temperature for an extended period of time to allow the Maillard reaction to occur between lactose and the milk proteins, during which the milk turns brown (Ni et al., 2017). The bacterial starter culture is then added to initiate the fermentation process after the Maillard reaction is complete. Apart from the color difference, this manufacturing process also leads to variations in taste, flavor, and nutritional value compared with other fermented products (Martins et al., 2000). These functional and sensorial differences occur due to chemical changes during the fermentation process, which result in milk metabolomic profiles that vary depending on the type of milk and the phase of fermentation. Fermented brown milk has good flavor, as demonstrated by Dong and Zhang (2005), who found the fermented brown milk product Yakult to have a unique aroma that is a balanced mixture of sour and sweet.

The metabolome contains a wide range of small molecules such as carbohydrates, lipids, organic acids, peptides, AA, nucleic acids, and vitamins that together represent a snapshot of metabolism (Zhao 
and Lin, 2014). Metabolomics is an emerging field within "omics" science. It aims to monitor changes in metabolite profiles using sophisticated analytical techniques. Currently, the main analytical techniques used are hyphenated techniques such as GC and HPLC coupled with MS, nuclear magnetic resonance, and near-infrared spectrometry (Mozzi et al., 2013). Liquid chromatography-MS is one of the most commonly used analytical platforms for metabolome analysis because of its high mass accuracy and resolution (Cheng et al., 2018). Mass spectrometry ${ }^{\text {Elevated Energy }}\left(\mathbf{M} \mathbf{S}^{\mathrm{E}}\right)$ is a new data collecting technique that offers 2 scanning functions to gather MS and tandem mass spectrometry (MS/MS) data simultaneously. In the first function, Q1, MS data are scanned for 50 to $1,000 \mathrm{~m} / z$ ions. In the second function, Q2, a normal high collision energy is used to break ions and scan the MS/MS ions. All ions enter into the quadrupole time-of-flight analyzer and are recorded by the detector (Wrona et al., 2005). The liquid chromatography-MS technology is used in multiple disciplines (e.g., pharmaceutical analysis, bioanalysis, and food science). In food science, metabolomic analysis has been used to evaluate food quality, processing safety, and the microbiology of raw materials and final products (Cevallos-Cevallos et al., 2009).

Traditionally, the species Streptococcus thermophilus and Lactobacillus bulgaricus are used as commercial fermented milk starter cultures. Streptococcus thermophilus is one of the most economically important species of lactic acid bacteria and is widely used in dairy fermentation due to its rapid rate of milk acidification. Moreover, it is also generally recognized as safe (Iyer et al., 2010). The strain S. thermophilus S10 was isolated from a naturally fermented milk product collected in Qinghai, China, by our laboratory staff. It has excellent fermentation characteristics including fast acid production. The fermented milk produced by S. thermophilus S10 is characterized by high viscosity and a unique aroma (Yao et al., 2018). The choice of S. thermophilus S10 for this study was based on previous experience with the strain in the production of fermented soybean milk, where it showed excellent fermentation characteristics (Guo et al., 2019). To the best of our knowledge, because fermented brown milk is an emerging new product, no studies have yet described changes in the metabolome during brown milk fermentation. Fei et al. (2019) described charcoal-fired yogurt as having a beautiful brown color and a flavor similar to toffee and caramel. Our goal in this study was to describe the metabolomic changes occurring during the brown milk fermentation process and identify metabolites that differed in abundance compared with brown milk. We used ultra-performance liquid chromatography coupled with a quadrupole-time-of-flight mass spectrometry ${ }^{\text {Elevated Energy }}$ (UPLC-QTOF $\mathbf{M S}^{\mathrm{E}}$ ). Differences between subgroups of samples were assessed by multivariate analysis, including principal component analysis and orthogonal partial least square-discriminate analysis (OPLS-DA). The findings of this study reveal the characteristics of fermented brown milk from a microbial metabolic perspective.

\section{MATERIALS AND METHODS}

\section{Materials and Equipment}

The S. thermophilus S10 strain used in this study was obtained from the Lactic Acid Bacteria Culture Collection, Inner Mongolia Agricultural University, China. The skim milk powder (containing 33\% protein) was purchased from Fonterra Co. Ltd., Auckland, New Zealand. Chromatography-grade reagents, including acetonitrile, formic acid, and ammonium hydroxide solution, were purchased from Sigma-Aldrich (St. Louis, $\mathrm{MO}$ ), and leucine-enkephalin was supplied by Waters (Manchester, UK). The taste sensor system SA402B was purchased from Insent (Atsugi-chi, Japan). The Acquity UPLC-Xevo G2 QTOF MS system and the MassLynx 4.1 Workstation and Progenesis QI software were purchased from Waters (Manchester, UK). Deionized water was produced by a Milli-Q water purification system (Millipore, Bedford, MA).

\section{Preparation of Brown Milk and Fermented Brown Milk}

Reconstituted milk was prepared by suspending the skim milk powder in sterilized water $(10 \% \mathrm{wt} / \mathrm{wt})$ and homogenizing it under high pressure $\left(65^{\circ} \mathrm{C}, 90 \mathrm{MPa}\right)$. Then the milk was heated to $95^{\circ} \mathrm{C}$ and incubated at this temperature in a thermostatically controlled water bath for $3 \mathrm{~h}$ for browning to occur. After the browning process, the milk was cooled to $42^{\circ} \mathrm{C}$ and the $S$. thermophilus S10 strain was inoculated into the brown milk at a concentration of $5 \times 10^{6} \mathrm{cfu} / \mathrm{mL}$, followed by incubation at $42^{\circ} \mathrm{C}$ until the $\mathrm{pH}$ of the milk reached 4.5 (i.e., when fermentation had ended). Samples were collected at $0 \mathrm{~h}$ (i.e., brown milk just added the starter culture) and at the end of fermentation. All samples were stored at $-80^{\circ} \mathrm{C}$ before analysis.

\section{Electronic Tongue Measurements}

The taste of brown milk and fermented brown milk were determined using the taste sensor system SA402B (Insent Inc.). Each sample was pretreated before measurement. Either $10 \mathrm{~g}$ of fermented brown milk (end of fermentation) or $10 \mathrm{~g}$ of brown milk as a reference (0 
time before fermentation) were sampled. Each sample was weighed accurately using an electronic balance and then diluted to $100 \mathrm{~g}$ with sterile distilled water. For taste evaluation samples were poured into a special beaker of the electronic tongue $(25 \mathrm{~mL}$ per sample volume) directly. Preliminary trials showed that the sensor fluctuated a lot at the beginning of the measurement. For this reason, each sample was measured 8 times; the first 5 readings were discarded and only the final 3 were used for statistical analysis.

\section{UPLC-QTOF MS ${ }^{E}$ Analysis}

Sample Preparation. Samples were each centrifuged at $12,000 \times g$ for $10 \mathrm{~min}$ at $4^{\circ} \mathrm{C}$ and the fat layer discarded. For each sample, $1 \mathrm{~mL}$ of supernatant was pipetted into a fresh centrifuge tube and mixed vigorously with $7 \mathrm{~mL}$ of acetonitrile for $2 \mathrm{~min}$. All samples were then centrifuged at $12,000 \times g$ for 10 min at $4^{\circ} \mathrm{C}$ to remove macromolecular aggregates like proteins (Mi et al., 2018). The supernatants were collected and concentrated by rotary evaporation for $10 \mathrm{~h}$ before reconstituting them in $500 \mu \mathrm{L}$ of acetonitrile solution (40\%). Samples were filtered through $0.22-\mu \mathrm{m}$ microporous membranes (Sigma-Aldrich) before being loaded onto the chromatographic column. A quality control (QC) sample was prepared by pooling equal volumes of all samples. The blank and the QC samples were first analyzed by chromatography to ensure good stability and repeatability of the chromatographic system. Then, a total of 24 milk supernatant samples [ 6 brown milk (0 time) and 6 fermented brown milk samples (end of fermentation) in the positive and negative ion mode] were randomly injected into the system; the QC sample was repeated after the injection of every 6 milk supernatant samples to control the stability of the instrument. The QC sample was analyzed 7 times in total to determine accuracy.

HPLC Conditions. Chromatographic analysis was done using a Waters ACQUITY UPLC system incorporating a $2.1 \times 100 \mathrm{~mm}$ Waters HSS T3 column. The flow rate was set at $0.45 \mathrm{~mL} / \mathrm{min}$, whereas the injection volume was $5 \mu \mathrm{L}$ per sample. All samples were eluted using a gradient mode. The positive ion mode mobile phases were $0.1 \%$ formic acid aqueous solution (A) and $0.1 \%$ formic acid containing acetonitrile solution (B). The gradient elution conditions of the positive ion mode were $0.25 \mathrm{~min}, 98 \% \mathrm{~A}, 2 \% \mathrm{~B} ; 12 \mathrm{~min}, 2 \% \mathrm{~A}, 98 \%$ B; 14 min, $2 \%$ A, $98 \%$ B; 14.10 min, $98 \%$ A, 2\% B; $17 \mathrm{~min}, 98 \% \mathrm{~A}, 2 \% \mathrm{~B}$. The negative ion mode mobile phases were $0.1 \%$ ammonium hydroxide (A) and acetonitrile solution (B). The gradient elution conditions for the negative ion mode were $3 \mathrm{~min}, 90 \% \mathrm{~A}, 10 \% \mathrm{~B}$; 6 min, $70 \%$ A, $30 \%$ B; 8 min, $50 \%$ A, $50 \%$ B; 12 min,
$40 \%$ A, $60 \%$ B; $14 \min , 10 \%$ A, $90 \%$ B; $15 \min , 10 \%$ A, $90 \% \mathrm{~B}$; and $16 \mathrm{~min}, 90 \% \mathrm{~A}, 10 \% \mathrm{~B}$. The total run time was $17 \mathrm{~min}$.

MS Conditions. The MS analysis was done on a QTOF MS (Waters MS Technologies, Manchester, UK) with an electrospray ionization source operating in positive and negative ionization modes. The desolvation gas flow rate was set to $800 \mathrm{~L} / \mathrm{h}$ at a temperature of $500^{\circ} \mathrm{C}$; the cone gas rate was set at $50 \mathrm{~L} / \mathrm{h}$; and the source temperature was set to $110^{\circ} \mathrm{C}$. The capillary voltage and the sample cone voltage were $3.0 \mathrm{kV}$ and $40 \mathrm{~V}$, respectively. To ensure the accuracy and reproducibility of the data, a concentration of 200 pmol of leucine enkephalin was used as the calibration solution (corresponding to $\mathrm{m} / z 556.2771$ for positive ion mode and $m / z 554.2615$ for negative ion mode). The $\mathrm{MS}^{\mathrm{E}}$ method was used to allow analyte detection through low and high energy functions. The low energy function corresponded to the MS full-scan data acquisition, whereas the high energy function recorded unspecific fragmentation of ions detected in the low energy function without loss of signal intensity in this function scan from 50 to $1,200 \mathrm{~m} / z$ (Plumb et al., 2006).

\section{Data Processing and Multivariate Statistical Analysis}

The $\mathrm{MS}^{\mathrm{E}}$ raw data were collected by the MassLynx 4.1 workstation and processed using Progenesis QI, including peak extraction, peak alignment, deconvolution, and normalization with default settings (default automatic sensitivity and without minimum peak width). Data files were imported to the EZinfo 3.0 software for multidimensional statistical analysis, such as OPLS-DA. A score plot of the principal components was used to visualize differences in metabolite profiles among samples. The OPLS-DA was performed to identify differentially abundant metabolites using the cut-off criteria: variable importance in projection $>1$; $P$-value $<0.05$ in pairwise comparison with the MannWhitney test; and a fold change of $>2$ between the 2 groups.

\section{Identification of Differentially Abundant Metabolites and Metabolic Pathway Analysis}

The metabolites of interest were identified by matching their profiles relating to accurate mass values, retention time, isotope peak matching, mass-to-charge ratio, and fragment information against different databases, including Chemspider (http://www.chemspider.com/), KEGG (https://www.kegg.jp/), and METLIN (https:/ /metlin.scripps.edu) using the Progenesis QI software. Differentially abundant metabolites were identified by MS/MS fragment information matching. All differen- 


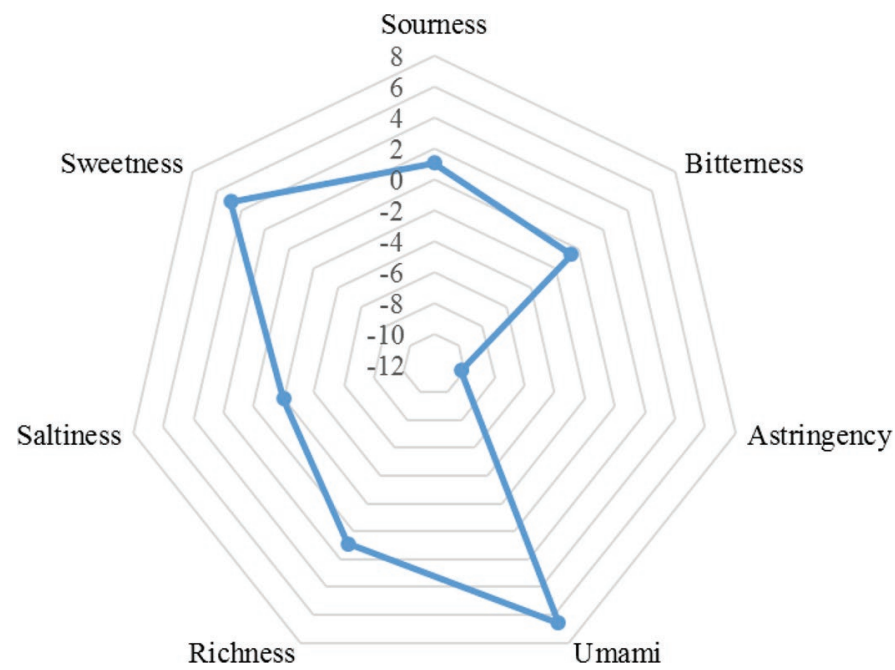

Figure 1. Radar chart of the taste of fermented brown milk as built by sensors.

tially abundant metabolites were searched against the KEGG database for metabolic pathway analysis.

\section{RESULTS AND DISCUSSION}

\section{Electronic Tongue Detection Results}

The average and standard deviation of the response signal values of the last 3 measurements of the sensor for fermented brown milk are shown in Table 1. A radar map of the taste of fermented brown milk was made based on the sensor signals (Figure 1). The sweet and umami taste of the fermented brown milk was particularly obvious; the sour taste was secondary; and bitterness, astringency, and salty tastes were hardly apparent (Figure 1). This was due to significant metabolic changes caused by $S$. thermophilus during fermentation, which produced metabolites that imparted taste to fermented brown milk.

\section{Identification of Metabolites that Were Differentially Abundant in Brown Milk and Fermented Brown Milk}

Typical UPLC-QTOF MS ${ }^{\mathrm{E}}$ base peak intensity chromatograms of brown milk and fermented brown milk are shown in Figure 2. Significant differences in the chromatographic/metabolite profiles between the 2 types of milk were apparent. Symbols representing the brown milk and fermented brown milk were distributed to different sides of the OPLS-DA score plot, respectively (Figure 3), suggesting that clear differences existed in the metabolomes of the 2 milks. To identify metabolites that were differentially abundant in the
2 milks, score plots from the OPLS-DA models were generated (Figure 4).

A total of 43 differentially abundant metabolites were identified (Table 2), including peptides, AA, carbohydrate metabolites, fatty acids and related metabolites, vitamins, and nucleosides.

\section{Major Metabolomic Differences Between Brown Milk and Brown Fermented Milk}

To visualize changes in the metabolite profile after fermentation, a heatmap was constructed to contrast the distribution of identified differential metabolites in brown milk and in fermented brown milk samples (Figure 5).

\section{Peptide and AA Metabolites}

Peptide content was generally higher in the fermented brown milk than in brown milk. Nineteen peptides were significantly more abundant in the fermented brown milk than in the brown milk (Table 2; Figure 5); these included Ala-Ser-Leu-Pro-Lys-Val-Tyr-Val, 2-OrnithylGly, Try-Pro-His, Gly-Gly, and Gly-Gly-Pro. Seven peptides were more abundant in brown milk than in fermented brown milk (Table 2; Figure 5).

The changes in the peptide profile following fermentation are likely to be due to the breakdown of milk proteins by the metabolic activity of $S$. thermophilus to acquire nutrients in the form of various oligopeptides (Larsen et al., 2006). Short peptides, such as Lys-GluSer-Thr-Leu, Ala-Ala-Pro-Thr, Leu-Lys-Leu, and LeuLys-Leu, have also been reported as enriched in the metabolite fingerprint of solidified yogurt (Wang et al., 2017). Fermented milk produced by a mixed starter culture of S. thermophilus and Lactobacillus plantarum generally contained a diversity of short peptides and AA, some of which are known to have functional attributes (Ghosh et al., 2013). Although our study did not directly investigate the functional or sensory properties of small peptides in fermented brown milk, many published studies have described the beneficial functions of short peptides derived from milk proteins

Table 1. Response signals of the electronic tongue sensors

\begin{tabular}{lc}
\hline Taste & Response signal $( \pm \mathrm{SD})$ \\
\hline Sourness & $-1.04 \pm 0.03$ \\
Bitterness & $0.63 \pm 0.07$ \\
Astringency & $-10.22 \pm 1.94$ \\
Umami & $6.56 \pm 0.01$ \\
Richness & $0.86 \pm 0.02$ \\
Saltiness & $1.98 \pm 0.00$ \\
Sweetness & $4.85 \pm 0.04$ \\
\hline
\end{tabular}




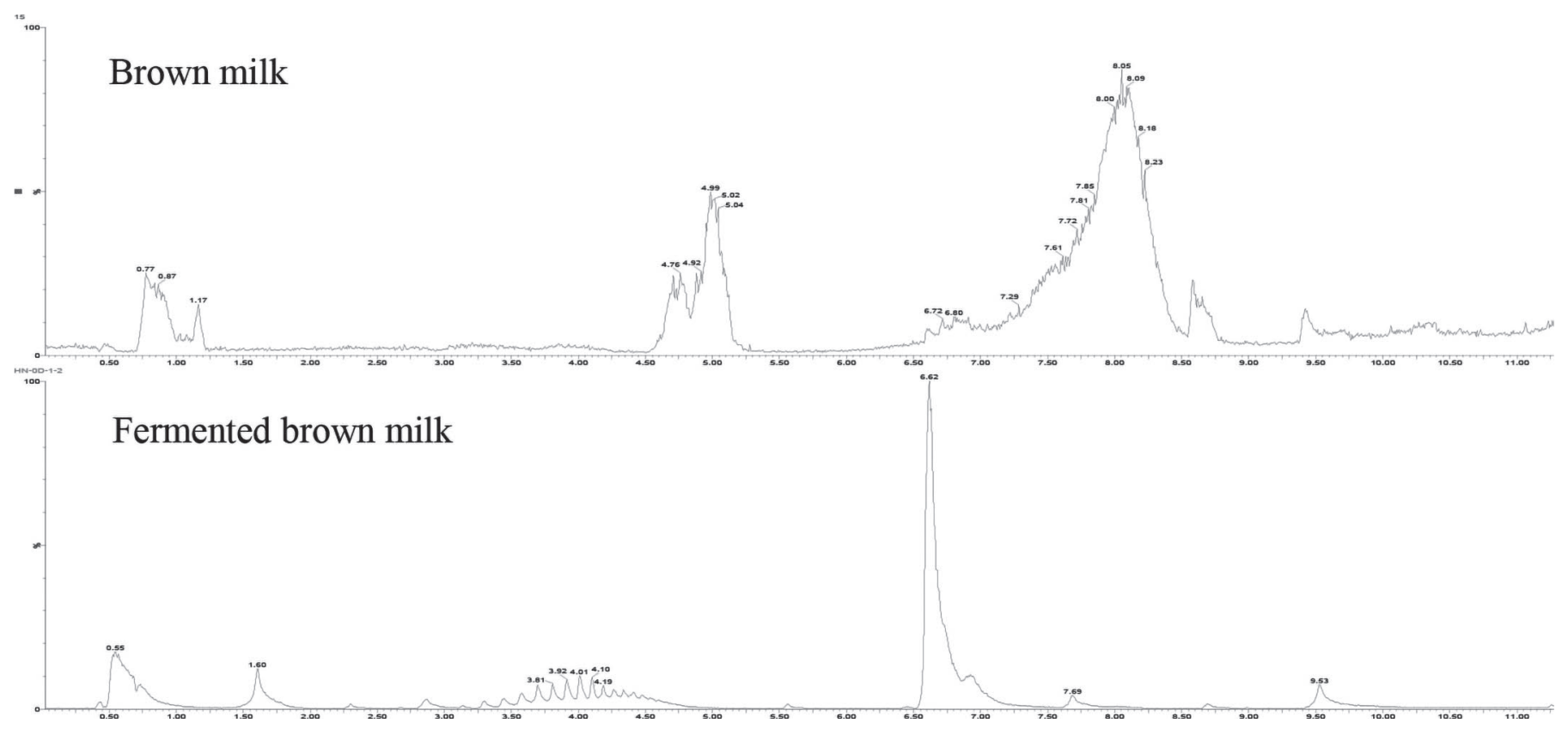

Figure 2. Base peak intensity chromatograms of brown milk ( $\mathrm{HN} 0 \mathrm{H})$ and fermented brown milk (HN 0D).

during fermentation. For example, studies have shown that these peptides can lower cholesterol, thus reducing hypertension, thrombotic risk, and antioxidation functions (Gibbs et al., 2004; Pak et al., 2008). To prove that the peptides in fermented brown milk also have these physiological functions requires further research.

In addition to desirable health-related functions, some of the peptides we identified in fermented brown milk are also known to influence the flavor of fermented products. For example, the dipeptides and tripeptides that contain the AA sequences Gly-Gly, Gly-Gly-Gly, and Gly-Gly-Pro are known to elicit a sweet taste (Kato et al., 1989), whereas those containing Asp-TrpAsp-Ser, Ala-Lys-Ser-Pro-Lys-Lys-Pro, and Gly-GlnAla-Arg elicit a sour taste (Kato et al., 1989). Peptides containing L-Orn-Gly-Gly-Gly-Orn-Gly-Gly-Orn-L-Orn and 2-Orn-Gly elicit a salty taste (Tuong and Philippossian, 1987), as does the dipeptide L-Orn-Tau, where the salty taste is positively correlated with the degree of dissociation of carboxyl or sulfonyl groups in the peptide (Tuong and Philippossian, 1987). The peptides Asp-Trp-Asp-Ser, Glu-Glu, Asp-Leu, and Glu-Leu are associated with the umami taste (Ohyama et al., 1988; Kato et al., 1989), which together contribute to the unique flavor of fermented brown milk. In addition to the increased abundance of short peptides in fermented brown milk, an accumulation of AA was also observed. The fermented brown milk had significantly more LLeu and L-Lys (2- and 276-fold enriched, respectively) compared with brown milk (Figure 5). These 2 AA are known to contribute a unique bitter taste to fermented products due to their hydrophobic side chain (Kato et al., 1989). These small peptides and AA could also be important components that confer the unique sensory characteristics and nutritional qualities of fermented brown milk.

\section{Fatty Acids and Related Metabolites}

Our results showed that fermentation of brown milk changed the levels of SFA (Table 2). Owing to the complexity of lipid metabolism and the wide diversity of metabolites, fatty acid metabolism has become a key focus in metabolomics research in recent years (Liu, 2017). Hermansson et al. (2011) mapped the biosynthesis and metabolism of glycerophospholipids in all mammalian cells and provided a comprehensive basis for studying lipid metabolism. According to Rao and Reddy (1984), whole milk fermented by S. thermophilus had higher levels of saturated and free fatty acids than unfermented whole milk; among the fatty acid metabolites, stearic acid and oleic acid levels increased the most, whereas monoglycerides disappeared completely during the fermentation process. Oleic acid and stearic acid are beneficial as they soften blood vessels and are thus likely to be health promoting (Calder, 1996).

Other than the beneficial health effects, fatty acid composition can also influence the taste, flavor, and texture of food. The main UFA in milk is oleic acid, accounting for about $70 \%$ of total UFA (Li et al., 2012). 


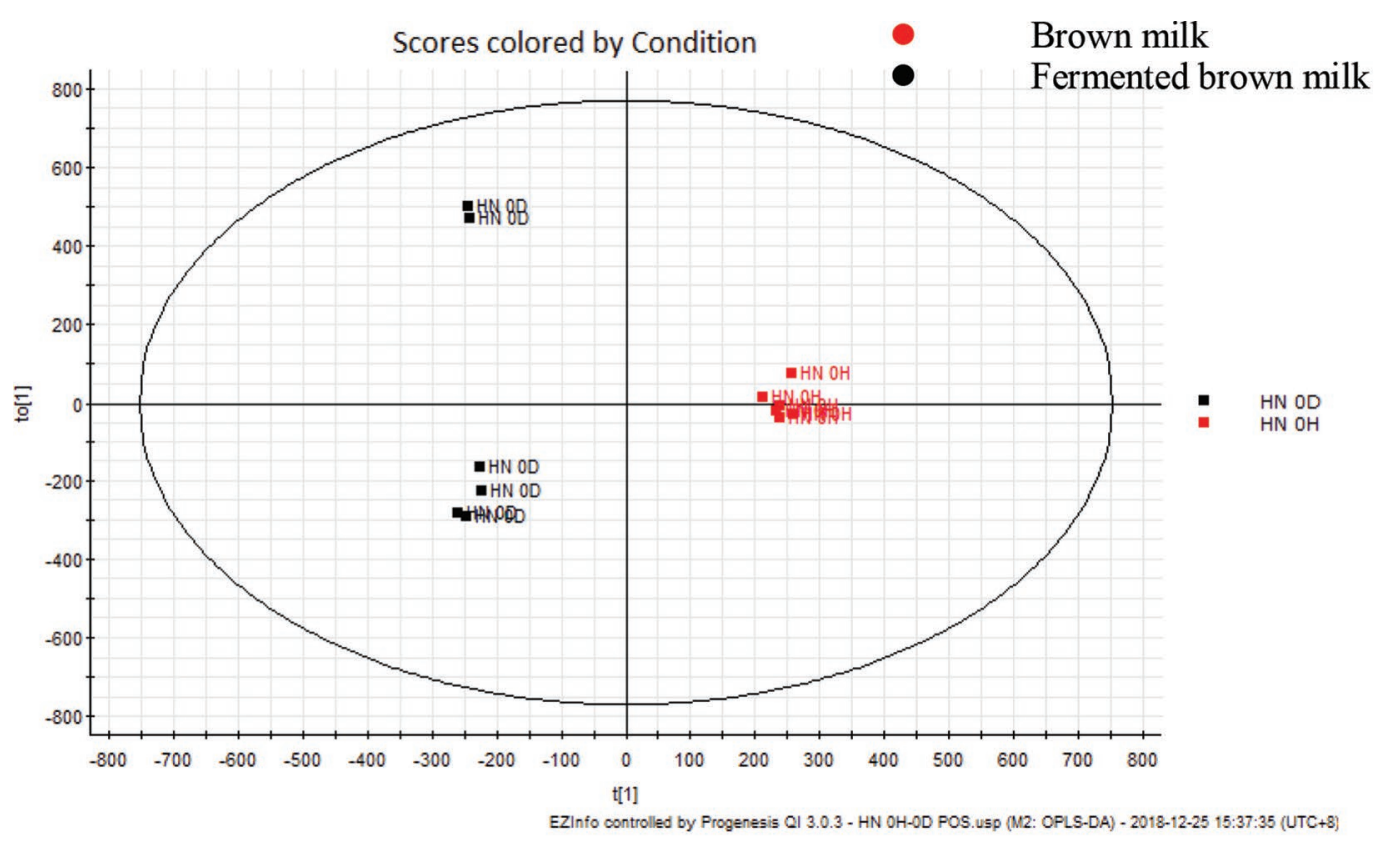

\section{$\mathrm{ESI}^{+}$}

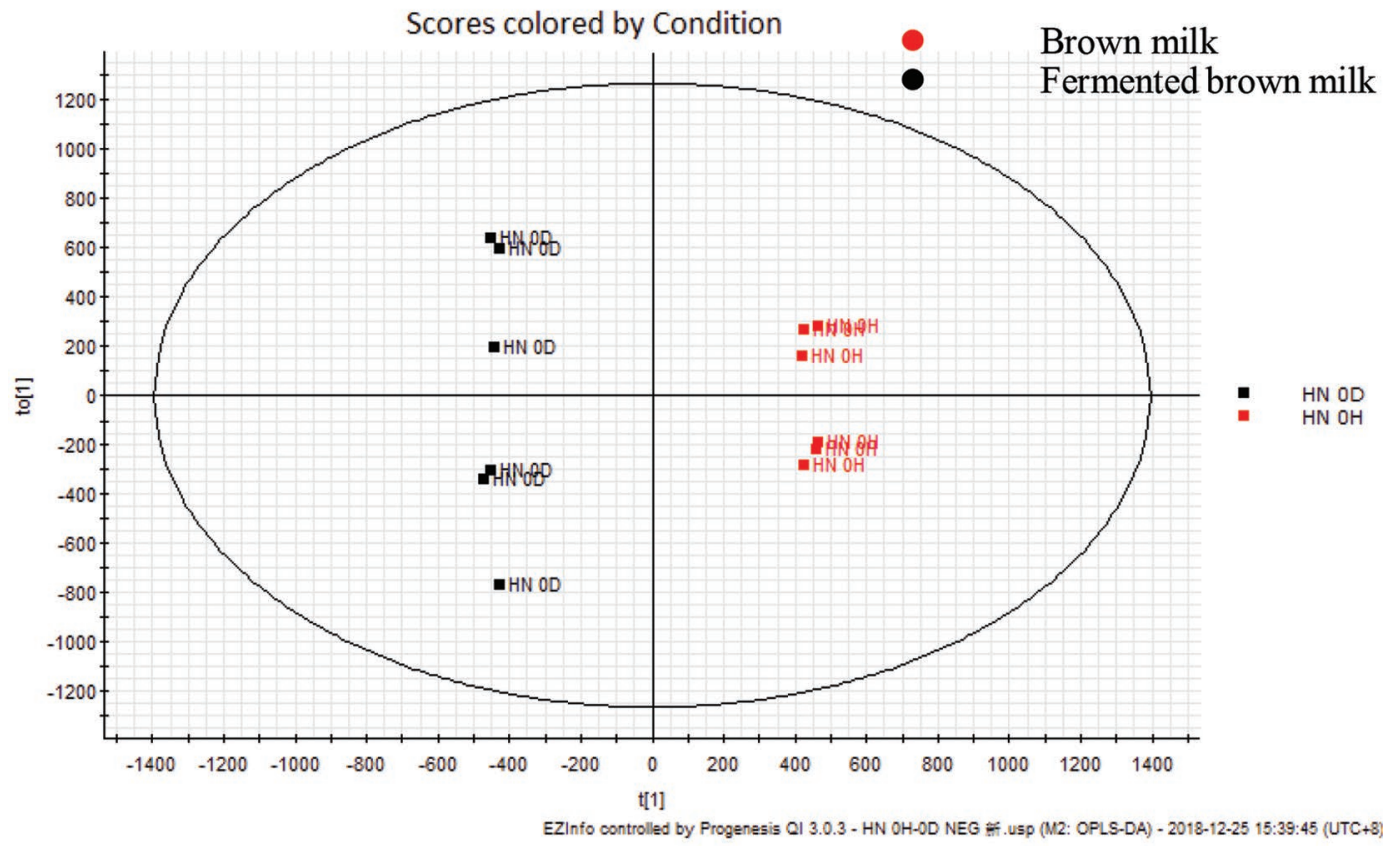

\section{$\mathrm{ESI}^{-}$}

Figure 3. Orthogonal partial least square-discriminate analysis (OPLS-DA) models of brown milk (HN 0H) and fermented brown milk (HN 0D). ESI = electrospray ionization; UTC = coordinated universal time. to[1] and $\mathrm{t}[1]$ are the 2 most important new variables in summarizing the data set. Progenesis QI software, Waters, Manchester, UK. 


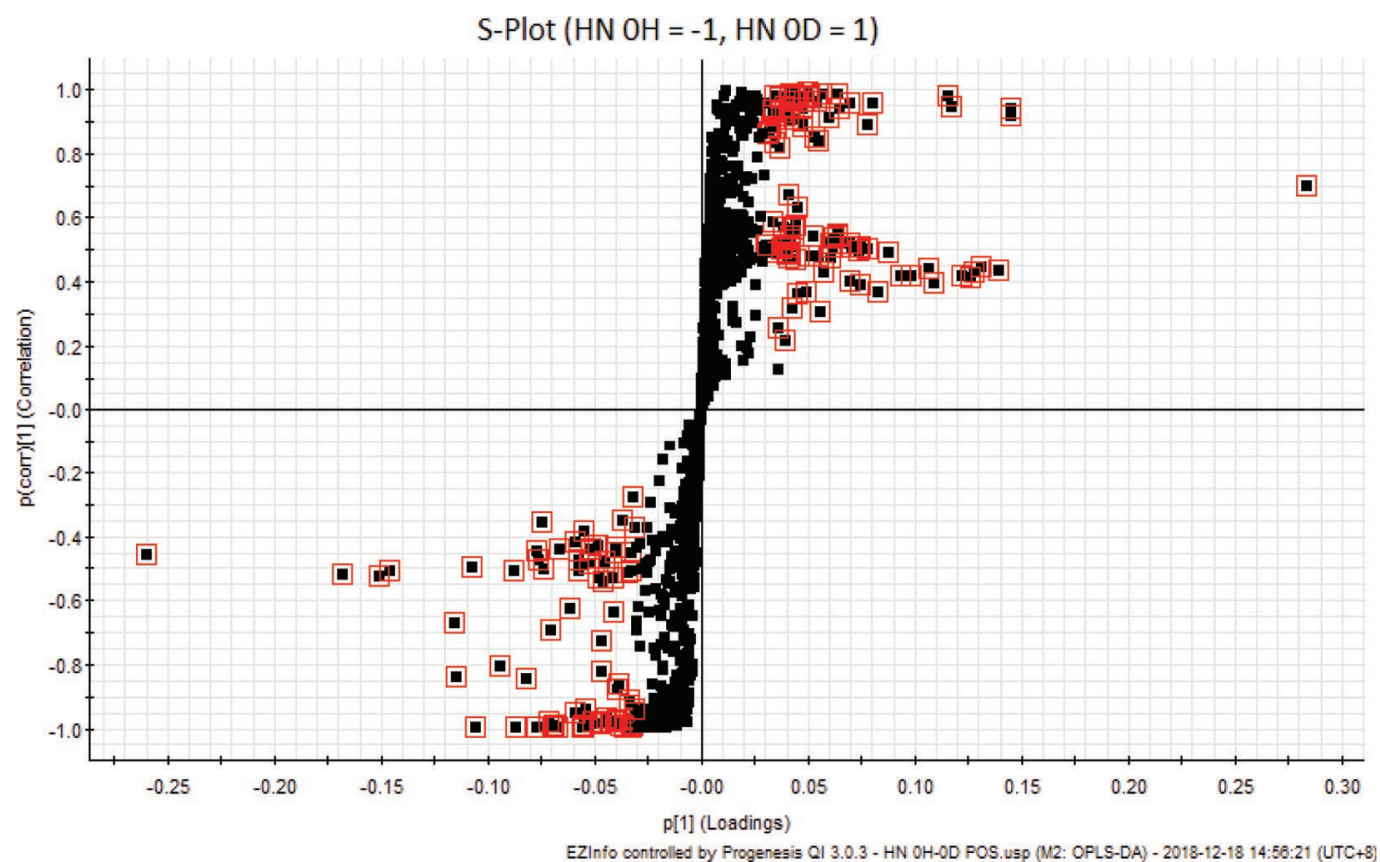

\section{$\mathrm{ESI}^{+}$}

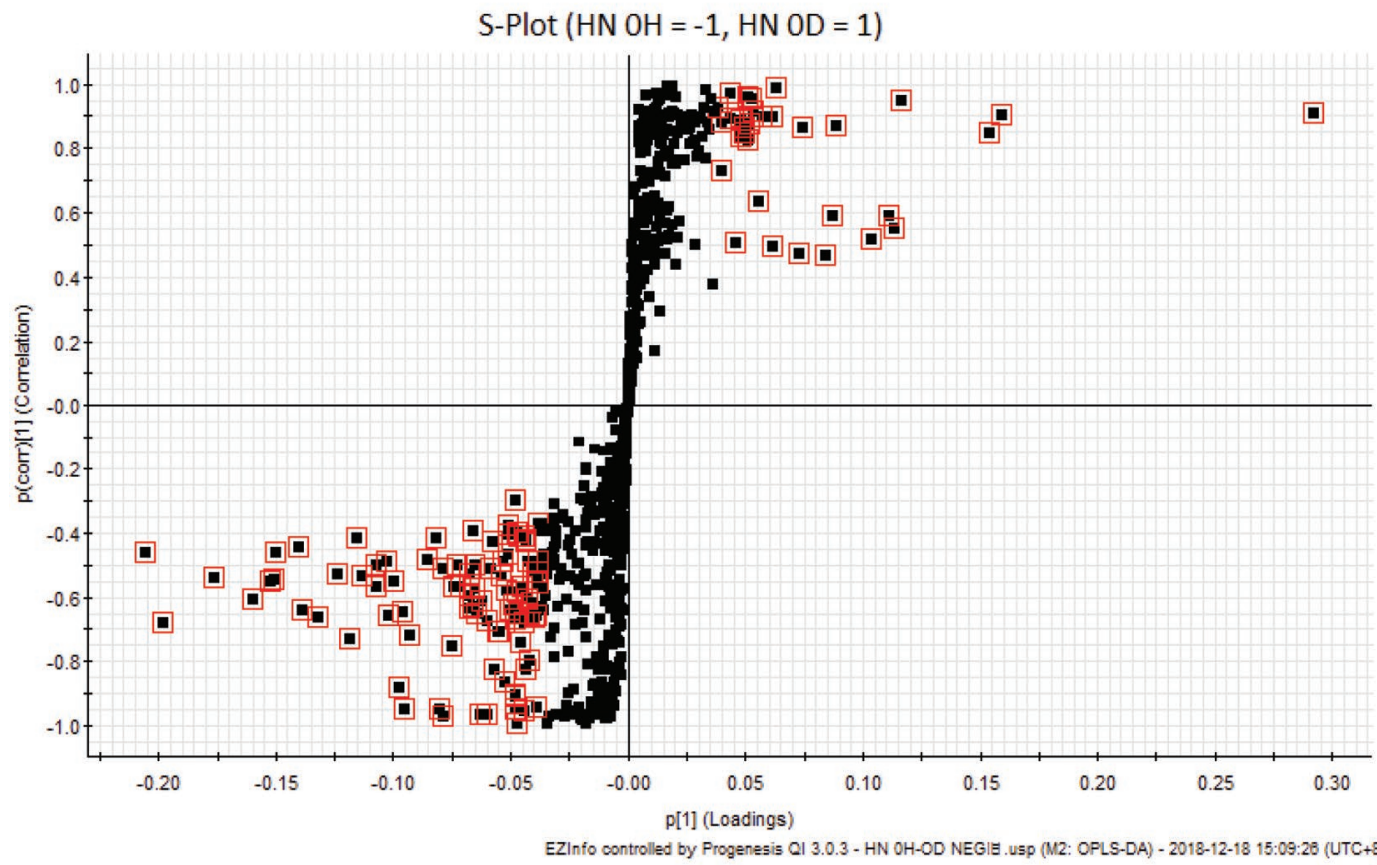

$\mathrm{ESI}^{-}$

Figure 4. Score plot (S-Plot) of brown milk ( $\mathrm{HN} 0 \mathrm{H})$ and fermented brown milk (HN 0D). Red squares indicate substances with mean variable importance in projection that are $>1$. This is used to identify differentially abundant metabolites. ESI $=$ electrospray ionization; UTC $=$ coordinated universal time. p[1] is $P$-value, a parameter used to determine the results of hypothesis testing. Progenesis QI software, Waters, Manchester, UK. 
Li et al.: METABOLITES IN FERMENTED BROWN MILK

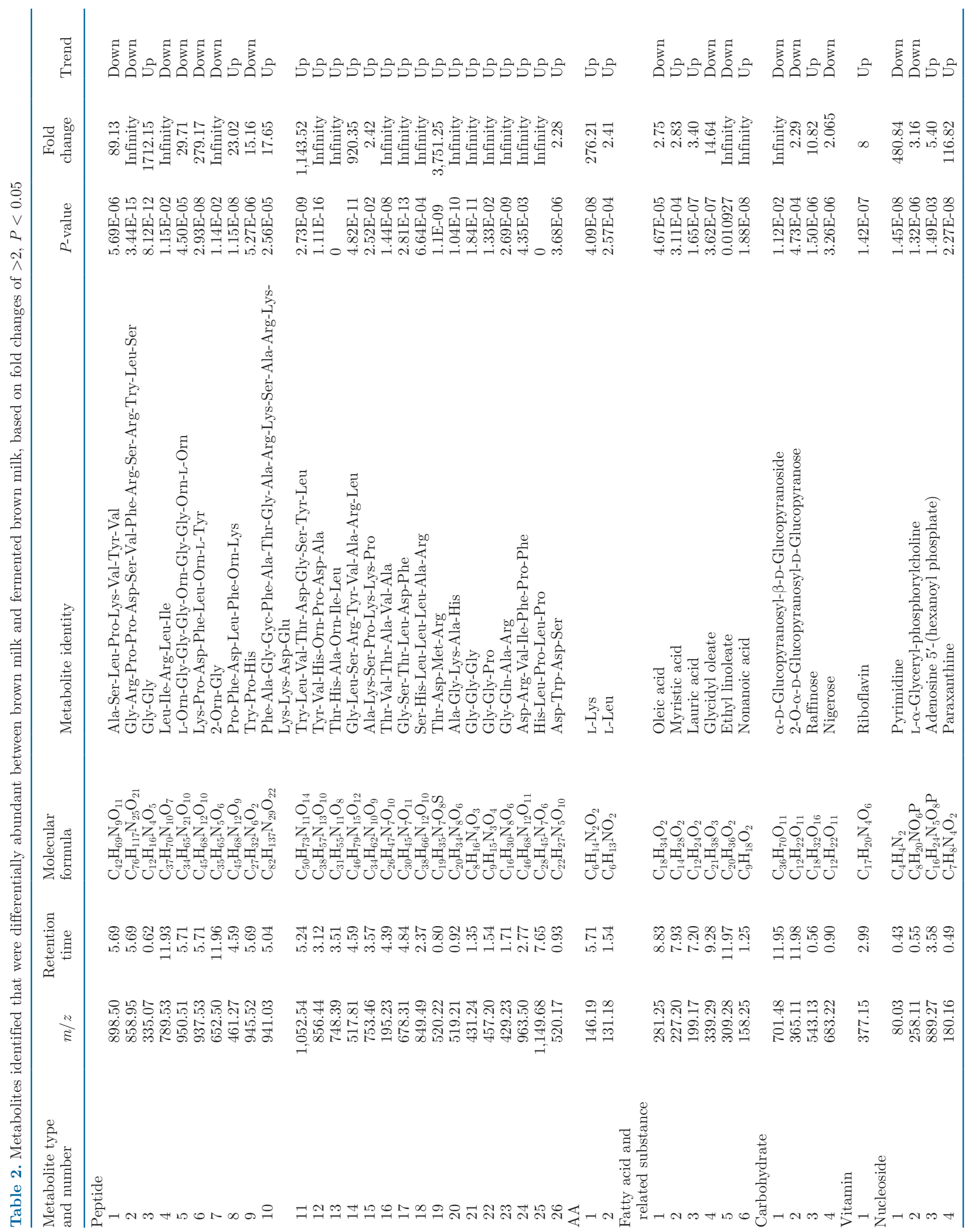



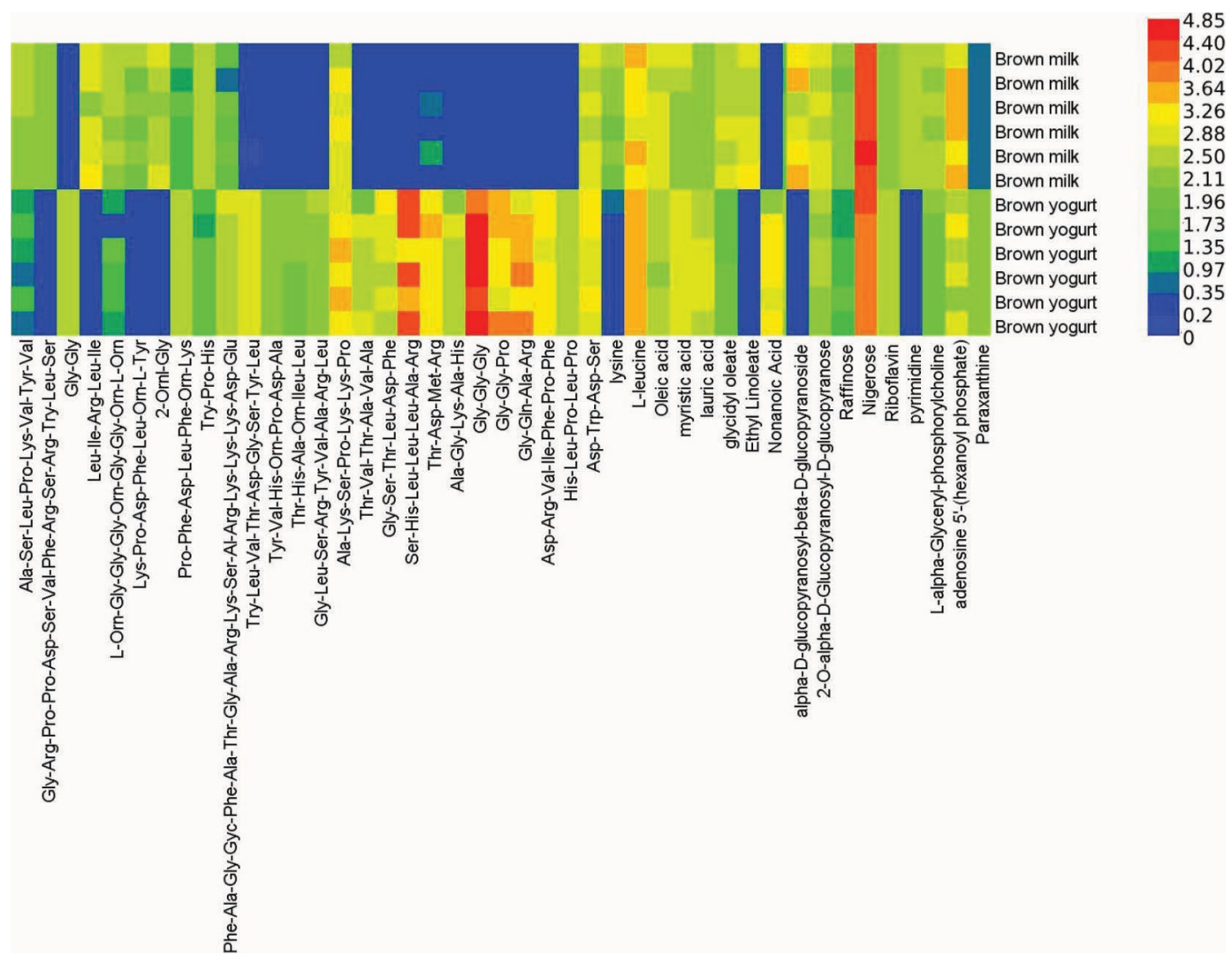

Figure 5. Heatmap showing relative quantities of metabolites that are differentially abundant in brown milk and fermented brown milk. The color of the box represents the relative quantity of that substance in each sample (blue indicates the lowest and red indicates the highest).

Attaie and Richter (1996) and Fan and Wang (2008) found that mature Cheddar cheese contained 12 free fatty acids and high levels of oleic acid and palm oil, which is consistent with our results. Free fatty acids can also can also be involved in a matrix of metabolic reactions related to aromatic and aroma substances. For example, S-methyl thioesters are produced from short-chain fatty acids and provide a unique flavor to fermented dairy products (Pinheiro et al., 2002). If the concentration of free fatty acids is reduced, typical flavors will be missing (Foda et al., 1974).

\section{Nucleoside Metabolites}

Our results showed that levels of pyrimidine and L- $\alpha$-glyceryl-phosphorylcholine were much lower in fermented brown milk than in brown milk (Figure 4). In contrast, adenosine 5'-hexanoyl phosphate and hypoxanthine accumulated during brown milk fermentation; their levels increased 5- and 116-fold by the end of fermentation (Figure 4). Pyrimidine is one of the most abundant metabolic species in cells, and it plays an important role in generating cellular energy and in cell signaling. Pyrimidine is used by $S$. thermophilus as a source of carbon, nitrogen, and energy for growth (Editorial Board of Dairy Science and Technology, 2016). Adenine is broken down by adenine deaminase to form hypoxanthine, which is further broken down into xanthine, and after a series of further transformations, glycine is finally formed (Editorial Board of Dairy Science and Technology, 2016). Although nucleosides are biologically important, they have less effect on the sensory quality of fermented products, especially the nonphosphorylated forms (Charpentier et al., 2005).

\section{Vitamins}

Levels of riboflavin (vitamin $\mathrm{B}_{2}$ ) in fermented brown milk were 8 times higher than in brown milk (Table 2 ); this is likely to be because $S$. thermophilus S10 produces riboflavin. Thus, selection of a suitable starter culture could increase the concentrations of specific water-soluble vitamins. It is known that some lactic acid bacteria, including Streptococcus species, can produce riboflavin. Synthesis of riboflavin by microbes relies on the presence of substrates such as guanosine triphosphate and D-ribulose 5-phosphate. The imidazole ring of guanosine triphosphate is first hydrolyzed to 
form a 4,5-diaminopyrimidine, which is then converted to 5-amino-6-ribitol amino-2,4 $(1 \mathrm{H} 3 \mathrm{H})$-pyrimidinedione by a series of deamination, chain reduction, and dephosphorylation. Condensation of 5-amino-6-ribitol amino-2,4 (1H 3H)-pyrimidinedione with 3,4-dihydroxy-2-butanone 4-phosphate from ribulose provides 6,7-dimethyl-8-ribityllumazine. Disproportionation of anhydrous theophylline derivatives produces riboflavin and 5-amino-6-ribitol amino-2,4 $(1 \mathrm{H} 3 \mathrm{H})$-pyrimidinedione, which circulates in the biosynthetic pathway (Editorial Board of Dairy Science and Technology, 2016). In the presence of riboflavin, the photo-oxidative decomposition of iso- $\alpha$-acid can produce an aged flavor. Therefore, an increased riboflavin concentration will likely affect the flavor, particularly the sour taste of brown fermented milk.

\section{Carbohydrates}

Carbohydrate metabolites such as $\alpha$-D-glucopyranosyl$\beta$-D-glucopyranoside, $\quad 2-\mathrm{O}-\alpha-\mathrm{D}$-glucopyranosyl-Dglucopyranose, raffinose, and nigerose were found in brown milk, and the levels of these carbohydrate metabolites changed after fermentation, which was likely to be due to the Maillard reaction and microbial metabolism. The level of 2-O- $\alpha$-D-Glucopyranosyl-Dglucopyranose was 2.29 times lower in fermented brown milk than brown milk. Pyran is the main structure of pyranose; simple sugars like glucose, mannose, galactose, and other hexoses exist mostly in the form of pyranose. At the early stage of the Maillard reaction, glucose and AA undergo a carbonyl ammonia condensation reaction. In the intermediate stage of the Maillard reaction, fructosylamine is deaminated and dehydrated to form hydroxymethylfurfural, which was detected in brown milk. Glycosides such as $\alpha$-D-glucopyranosyl$\beta$-D-glucopyranoside and 2-O- $\alpha$-D-glucopyranosyl-Dglucopyranose taste sweet, which may contribute, at least in part, to the sweetness of fermented brown milk.

\section{CONCLUSIONS}

This study compared the metabolomes of brown milk and fermented brown milk. Significant changes were observed in the abundance of various metabolites after fermentation including peptides, AA, carbohydrates, fatty acids, vitamins, and nucleosides. Some of these changes could contribute to the unique sensorial quality of fermented brown milk. In particular, some of the peptides could contribute to bitterness and astringency, whereas carbohydrates could contribute to the sweetness of the product. The current data have provided basic information on the metabolite profiles of brown milk and fermented brown milk, as well as preliminary identification of metabolites that are differentially abundant in brown milk and fermented brown milk. Such data are valuable references for future development and improvement of brown milk fermentation products.

\section{ACKNOWLEDGMENTS}

This research was funded by the National Natural Science Foundation of China, Hohhot (Grant No. 31771954). We have no conflicts of interest.

\section{REFERENCES}

Attaie, R., and R. L. Richter. 1996. Formation of volatile free fatty acids during ripening of Cheddar-like hard goat cheese. J. Dairy Sci. 79:717-724. https://doi.org/10.3168/jds.S0022-0302(96)76418-4.

Calder, P. C. 1996. Effects of fatty acids and dietary lipids on cells of the immune system. Proc. Nutr. Soc. 55(1B):127-150. https://doi .org/10.1079/PNS19960015.

Cevallos-Cevallos, J. M., J. I. Reyes-De-Corcuera, E. Etxeberria, M. D. Danyluk, and G. E. Rodrick. 2009. Metabolomic analysis in food science: A review. Trends Food Sci. Technol. 20:557-566. https://doi.org/10.1016/j.tifs.2009.07.002.

Charpentier, C., J. Aussenac, M. Charpentier, J.-C. Prome, B. Duteurtre, and M. Feuillat. 2005. Release of nucleotides and nucleosides during yeast autolysis: Kinetics and potential impact on flavor. J. Agric. Food Chem. 53:3000-3007. https://doi.org/10.1021/ jf040334y.

Cheng, J., W. Lan, G. Zheng, and X. F. Gao. 2018. Metabolomics: A high-throughput platform for metabolite profile exploration. Methods Mol. Biol. 1754:265-292. https://doi.org/10.1007/978-1 -4939-7717-8_16.

Dong, T., and G. S. Zhang. 2005. Discuss on the technology of Yakult flavored lactobacillus drink. Zhongguo Rupin Gongye 33:40-41.

Editorial Board of Dairy Science and Technology. 2016. State Key Laboratory of Dairy Biotechnology, Fermented milk. Chemical Industry Press, China.

Fan, S. L., and M. Y. Wang. 2008. Gas chromatography-mass spectrometry for free fatty acids varieties and content of ripening Cheddar cheese. Food Res Dev. 29:122-123.

Fei, Y. M., G. S. Zhang, Z. X. Liu, and X. Y. Wang. 2019. Production technology and optimal packaging of carbonized yogurt with direct vat starter. Pack Eng. 40:46-53.

Foda, E. A., E. G. Hammond, G. W. Reinbold, and D. K. Hotchkiss. 1974. Role of fat in flavor of Cheddar cheese. J. Dairy Sci. 57:11371142. https://doi.org/10.3168/jds.S0022-0302(74)85026-5.

Ghosh, D., D. K. Chattoraj, and P. Chattopadhyay. 2013. Studies on changes in microstructure and proteolysis in cow and soy milk curd during fermentation using lactic cultures for improving protein bioavailability. J. Food Sci. Technol. 50:979-985. https://doi .org/10.1007/s13197-011-0421-1.

Gibbs, B. F., A. Zougman, R. Masse, and C. Mulligan. 2004. Production and characterization of bioactive peptides from soy hydrolysate and soy-fermented food. Food Res. Int. 37:123-131. https:// doi.org/10.1016/j.foodres.2003.09.010.

Guo, S., Z. H. Han, T. Huang, Y. Zheng, Y. J. Wang, M. Bai, J. C. Wang, T. S. Sun, and H. P. Zhang. 2019. Application of Streptococcus thermophilus S10 compound Lactobacillus plantarum P-8 in fermented soymilk. Food Fer. Ind. 45:11-17.

Hermansson, M., K. Hokynar, and P. Somerharju. 2011. Mechanisms of glycerophospholipid homeostasis in mammalian cells. Prog. Lipid Res. 50:240-257. https://doi.org/10.1016/j.plipres.2011.02.004.

Iyer, R., S. K. Tomar, T. U. Maheswari, and R. Singh. 2010. Streptococcus thermophilus strains multifunctional lactic acid bacteria. Int. Dairy J. 20:133-141. https://doi.org/10.1016/j.idairyj.2009.10 .005 . 
Kato, H., M. R. Rhue, and T. Nishimura. 1989. Role of free amino acids and peptides in food taste. ACS Symp. 388:158-174. https:/ /doi.org/10.1021/bk-1989-0388.ch013.

Larsen, N., M. Boye, H. Siegumfeldt, and M. Jakobsen. 2006. Differential expression of proteins and genes in the lag phase of Lactococcus lactis ssp. lactis grown in synthetic medium and reconstituted skim milk. Appl. Environ. Microbiol. 72:1173-1179. https://doi .org/10.1128/AEM.72.2.1173-1179.2006.

Li, Y. L., Z. P. Yang, L. L. Chang, D. J. Ji, X. L. Wang, Z. H. Gan, S. Liu, and X. H. Liu. 2012. The improvement of gas chromatography analysis of composition in milk fatty acid. China Cattle Sci. $38: 18-21$.

Liu, G. L. 2017. A lipid metabolomics study of pancreatic cancer based on UPLC-TOF/MS approach. PhD Diss. Zhengzhou University, Henan, China.

Loveday, S. M., A. Sarkar, and H. Singh. 2013. Innovative yoghurts: Novel processing technologies for improving acid milk gel texture. Trends Food Sci. Technol. 33:5-20. https://doi.org/10.1016/j.tifs 2013.06.007.

Ma, Z. M., S. X. Chu, Y. F. Kang, and J. Q. Cao. 2016. Development of a brown yoghurt. Anhui Nongye Kexue 44:90-93., 98.

Martins, S. I. F. S., W. M. F. Jongen, and M. A. J. S. van Boekel. 2000. A review of Maillard reaction in food and implications to kinetic modelling. Trends Food Sci. Technol. 11:364-373. https:// doi.org/10.1016/S0924-2244(01)00022-X.

Mi, Z. H., L. Y. Kwok, J. G. Xue, Y. N. Wang, H. P. Zhang, and Y. F. Chen. 2018. Fermentation dynamics of Lactobacillus helveticus H9 revealed by ultra-performance liquid chromatography quadrupole time-of-flight mass spectrometry. Int. J. Food Sci. Technol. $53: 1442-1451$

Mozzi, F., M. E. Ortiz, J. Bleckwedel, L. De Vuyst, and M. Pescuma. 2013. Metabolomics as a tool for the comprehensive understanding of fermented and functional foods with lactic acid bacteria. Food Res. Int. 54:1152-1161. https://doi.org/10.1016/j.foodres.2012.11 .010 .

Ni, D., L. Li, H. B. Zhang, and G. W. Zhang. 2017. Development of brown drinking yoghurt. Zhongguo Rupin Gongye 45:56-58.

Ohyama, S., N. Ishibashi, and M. Tamura. 1988. Synthesis of bitter peptides composed of aspartic acid and glutamic acid. J. Agric. Chem. Soc. Japan. 52:871-872.

Pak, V. V., M. Koo, M. J. Kim, H. J. Yang, L. Yun, and D. Y. Kwon. 2008. Modeling an active conformation for linear peptides and de- sign of a competitive inhibitor for HMG-CoA reductase. J. Mol. Recognit. 21:224-232. https://doi.org/10.1002/jmr.889.

Pinheiro, R., I. Belo, and M. Mota. 2002. Oxidative stress response of Kluyveromyces marxianus to hydrogen peroxide, paraquat and pressure. Appl. Microbiol. Biotechnol. 58:842-847. https://doi .org/10.1007/s00253-001-0927-y.

Plumb, R. S., K. A. Johnson, P. Rainville, B. W. Smith, I. D. Wilson, J. M. Castro-Perez, and J. K. Nicholson. 2006. UPLC/MS(E): A new approach for generating molecular fragment information for biomarker structure elucidation. Rapid Commun. Mass Spectrom. 20:1989-1994.

Rao, D. R., and J. C. Reddy. 1984. Effects of lactic fermentation of milk on milk lipids. J. Food Sci. 49:748-750. https://doi.org/10 $.1111 /$ j.1365-2621.1984.tb13201.x.

Tuong, H. B., and G. Philippossian. 1987. Alleged salty taste of Lornithyltaurine monohydrochloride. J. Agric. Food Chem. 35:165168. https://doi.org/10.1021/jf00073a036.

Wang, Y. N., X. M. Xi, Z. H. Mi, and T. S. Sun. 2017. Study on metabolic profile of set yoghurt based UPLC-Q-TOF MS ${ }^{\mathrm{E}}$. Zhongguo Rupin Gongye 45:4-7.

Wrona, M., T. Mauriala, K. P. Bateman, R. J. Mortishire-Smith, and D. O'Connor. 2005. 'All-In-One' analysis for metabolite identification using liquid chromatography/hybrid quadrupole time-of-flight Mass Spectrometry with collision energy switching. Rapid Commun. Mass Spectrom. 19:2597-2602. https://doi.org/10.1002/rcm 2101.

Yao, G. Q., S. D. Qimuge, and P. F. Gao. 2018. A strain of Streptococcus thermophilus and its application. Patent no. 109266581A. China.

Zhao, Y. Y., and R. C. Lin. 2014. UPLC-MS(E) application in disease biomarker discovery: The discoveries in proteomics to metabolomics. Chem. Biol. Interact. 215:7-16. https://doi.org/10.1016/j .cbi.2014.02.014.

\section{ORCIDS}

DanYang Li @ https://orcid.org/0000-0002-4008-4136

Lai-yu Kwok @ https://orcid.org/0000-0001-8791-1269

WenYi Zhang ํㅜ https://orcid.org/0000-0001-5530-4210 